\title{
Evaluating Structural Connectomics in Relation to Different Q-space Sampling Techniques
}

\author{
Paulo Rodrigues ${ }^{1,2}$, Alberto Prats-Galino ${ }^{3}$, David Gallardo-Pujol ${ }^{2}$, \\ Pablo Villoslada ${ }^{4}$, Carles Falcon ${ }^{5}$, and Vesna Prčkovska ${ }^{4}$ \\ 1 Mint Labs S.L., Barcelona, Spain \\ 2 Dept. of Personality, Faculty of Psychology, UB, Barcelona, Spain \\ 3 LSNA, Facultat de Medicina, UB, Barcelona, Spain \\ ${ }^{4}$ Center for Neuroimmunology, Department of Neurosciences, IDIBAPS, Hospital \\ Clinic, Barcelona, Spain \\ 5 Medical Imaging Platform, IDIBAPS, Barcelona, Spain
}

\begin{abstract}
Brain networks are becoming forefront research in neuroscience. Network-based analysis on the functional and structural connectomes can lead to powerful imaging markers for brain diseases. However, constructing the structural connectome can be based upon different acquisition and reconstruction techniques whose information content and mutual differences has not yet been properly studied in a unified framework. The variations of the structural connectome if not properly understood can lead to dangerous conclusions when performing these type of studies. In this work we present evaluation of the structural connectome by analysing and comparing graph-based measures on real data acquired by the three most important Diffusion Weighted Imaging techniques: DTI, HARDI and DSI. We thus come to several important conclusions demonstrating that even though the different techniques demonstrate differences in the anatomy of the reconstructed fibers the respective connectomes show variations of $20 \%$.
\end{abstract}

\section{Introduction}

Over the last decade, the study of complex networks has expanded dramatically across different scientific fields including Neuroscience. The brain is a complex system whose complex components continuously create complex patterns. Therefore, a natural paradigm for studying the brain is via network analysis. A comprehensive map of neural connections of the brain is called "connectome" [12]. At the macroscopic scale, the connectome can be seen as a network, usually represented as a matrix, where each vertex represents well-defined cortical or sub-cortical structures and the edges quantify the structural white matter connectivity as measured with tractography. When this matrix is estimated from Diffusion Weighted MRI (dwMRI) data we speak about structural connectivity. In the process of calculating the connectomes, from the measured data, several parameters are involved that can lead to variations of the connectivity matrices. In the structural connectome however, the most important step is the fibre tractography that depends not only

K. Mori et al. (Eds.): MICCAI 2013, Part I, LNCS 8149, pp. 671-678, 2013.

(C) Springer-Verlag Berlin Heidelberg 2013 
on the acquired dwMRI data but also on the choice for particular reconstruction and fibre-tracking algorithms, as pointed out by Bastiani et al [3]. The most common acquisition model is the Diffusion Tensor Imaging (DTI) [4] which requires modest q-space acquisitions with short scanning time ( 3-5min). However, DTI has been proved to have limitations in complex fibre areas. Therefore, more complex acquisitions models were developed, known as High Angular Resolution Diffusion Imaging (HARDI) [5], with denser q-space sampling on a spherical shell. It has been demonstrated that it is able to give good results even at lower (clinically preferable) b-values [6] resulting in scanning times of about $15 \mathrm{~min}$. The richest q-space sampling technique is Diffusion Spectrum Imaging [7], resolving more complex fibre configurations, however, at a cost of very long acquisition times ( $>35 \mathrm{~min})$. With the arrival of the connectomics many groups have initiated research in this direction, choosing the dwMRI data acquisition technique, however, without knowing how this choice influences the quality of the connectomes. The choice of suitable technique with high reproducibility for the purpose of constructing structural connectomics has not yet been properly addressed in literature, even though some attempts have been done for the functional connectome [8]. In this work, we evaluate the information difference contained in the structural connectomes constructed from the same subject scanned with different dwMRI acquisition techniques (DTI, HARDI and DSI). Since the goal is not evaluating all the possible parameters involved in the process, we acquire the data with the most commonly used parameters in literature. We perform the connectome construction for all the modalities with equal parameter settings. We furthermore test the reproducibility of the connectome and information difference in relation to the acquisition using network analysis. Finally we perform a 'blind' qualitative analysis of different fibre bundles involved in the connectome construction by an experienced Neuroanatomist.

\section{Methods}

Data: MRI acquisitions were performed on 5 healthy volunteers ( 4 male and 1 female, age:31.2 \pm 2.9 years) using a twice refocused spin-echo echo-planar imaging sequence on a $3 \mathrm{~T}$ Siemens Trio MRI scanner (Erlangen, Germany). Informed consent was obtained prior to the acquisition. The MRI protocol included the following sequences: a) 3D structural T1-weighted MPRAGE sequence: Repetition Time (TR): 1900ms, Echo Time (TE): $4.44 \mathrm{~ms}$, Inversion recovery time (TI): $1050 \mathrm{~ms}$, Flip angle: $8^{\circ}$, FOV: $220 \times 220 \mathrm{~mm}^{2}$, isometric $1 \mathrm{~mm}^{3}$; b) The parameters for the dwMRI sequences are given in the table 1 (top). We have acquired in total 21 datasets from which 7 DTI, 6 HARDI and 8 DSI. For some of the subjects the scans were repeated in the same scanning session or after one month. In table 1 (bottom) we report the subset of data we used for each of the performed tests and the number of subjects involved.

Connectome Construction: We calculated the connectomes using publicly available software, the connectome mapper $(\mathrm{cmp})^{1}[9]$. For all imaging modalities

\footnotetext{
${ }^{1}$ http://www.connectomics.org
} 
Table 1. Top: Scanning parameters used in our dwMRI acquisitions. * Due to technical reasons two DTI datasets were obtained using spatial resolution of $1.25 \times 1.25 \times 2.5 \mathrm{~mm}^{3}$. ${ }^{* *}$ Due to technical reasons one DSI dataset was obtained using spatial resolution of $3 \mathrm{~mm}^{3}$ isometric. bottom: Data overview per performed test.

\begin{tabular}{lcccc} 
Modality & DTI & HARDI & DSI & \\
\hline b-val $\left(\mathrm{s} / \mathrm{mm}^{2}\right)$ & 1000 & 1500 & $b_{\max }=8000$ & \\
num. grad. & 30 & 82 & 515 & \\
num. b $_{0}$ & 1 & 6 & $\mathrm{~N} / \mathrm{A}$ & \\
spatial res. $\left(\mathrm{mm}^{3}\right)$ & $2.5 \times 2.5 \times 2.5^{*}$ & $1.25 \times 1.25 \times 2.5$ & $2.2 \times 2.2 \times 3^{* *}$ & \\
TR/TE & $6900 / 89$ & $7600 / 98$ & $8200 / 164$ & \\
acq.time $($ min $)$ & 3.56 & 11.33 & 35.42 & \\
Evaluation & DTI & HARDI & DSI & \# subjects \\
\hline reproducibility & 5 & 4 & 6 & 3 \\
intra-subject & 7 & 6 & 6 & 4 \\
network based & 7 & 6 & 8 & 5 \\
qualitative & 3 & 3 & 3 & 3
\end{tabular}

we used the default settings (re-sampling the dwMRI data to $1 \mathrm{~mm}^{3}$ isometric voxel size using trilinear interpolation, tracking stopping criteria at angle $=60^{\circ}$, number of seeds $=32$, fibre filtering with enabled spline filter and cut-off filter in the interval of $[20 ; 500] \mathrm{mm})$. For DTI fibre tracking, the cmp uses the standard FACT method and for Qball and DSI FACT-alike algorithm implemented in the Diffusion Toolkit [10]. For two subjects to improve the registration step we performed non-linear registration using the T2 data, and for the rest of the subjects we used linear registration. We employed Lausanne parcellation since it offers 5 hierarchical scales to test and compare the quality of the connectomes. Depending on the imaging modality, DTI, HARDI (Qball) or DSI reconstruction was performed. For network creation, we first apply an absolute threshold in order to discard edges with less than 10 fibres (considered spurious fibres from data observation), and connection matrices are created by either binarizing edge weights, or by normalizing the edge weights with maximum number of found fibres.

Indices for Connectome Comparison and Quality Assessment: The simplest way of comparing networks is to assess the difference between their overall matrix representations. We computed the correlation between the graphs as described in Table 2. We computed several other indices (normalized rootmean-square deviation, dot product of the direct embedding of the matrix into a vector-space representation, Hamming distance and Fleiss' kappa reliability of agreement) but since they do not give further insight, we omit them for simplicity of presentation.

Table 2. Correlation measure of structural connectome agreement, represented as a matrix where $A_{i, j}$ is the weighted edge between nodes $i$ and $j$

$$
\begin{array}{ll}
\text { covariance } & \operatorname{cov}\left(\mathbf{y}_{\mathbf{1}}, \mathbf{y}_{\mathbf{2}}\right)=\frac{1}{N(N-1)} \sum_{i \neq j}^{N}\left(A_{i, j}^{(1)}-m_{1}\right)\left(A_{i, j}^{(2)}-m_{2}\right) \\
& \text { where } m_{l}=\frac{1}{N(N-1)} \sum_{i \neq j}^{N} A_{i, j}^{(l)} \\
\hline \text { correlation [11] } & \rho\left(\mathbf{y}_{\mathbf{1}}, \mathbf{y}_{\mathbf{2}}\right)=\frac{\operatorname{cov}\left(\mathbf{y}_{\mathbf{1}}, \mathbf{y}_{\mathbf{2}}\right)}{\sqrt{\operatorname{var}\left(\mathbf{y}_{\mathbf{1}}\right) \operatorname{var}\left(\mathbf{y}_{\mathbf{2}}\right)}}
\end{array}
$$


Network-Based Indices: To compare certain network features of the matrices we used the brain connectivity toolbox 2. We considered graph density, node strength, characteristic path length and global efficiency over binary undirected graph representation of the connectomes.

Track-Based Quality Assessment Indices: We extracted 40 fibre bundles that connect different cortical regions in the lowest parcellation scale (33) since it corresponds to the underlying anatomy. The regions were carefully selected spanning the whole brain in order to capture fibres with different neuroanatomical nature: commisural, projection, u-fibres and subcortical fibres. We exported the fibres of each reconstruction technique per subject and grouped in triplets of bundles visualised in the Amira 3 software. This data was presented to a professor in Neuroanatomy in a completely anonymized way (the fibres were only shown with different colors and no reference to the underlying acquisition modality). He was asked to score the accuracy of the fibres to the underlying anatomy as objectively as possible with scores from 1-3, where 3 stands for the most accurate and 1 for the least accurate technique. In case few techniques present similar accuracy they could be scored with the same score. We additionally report percentage of the missing fibre bundles (out of the 40 considered) that each technique fails to reconstruct, i.e., finds no fibers between 2 regions or only aberrant fibers.

\section{Results}

Reproducibility of the Structural Connectome: Structural connectome can be seen as a potential imaging marker sensitive to certain pathologies of the white matter (e.g., Multiple Sclerosis, Schizophrenia). If the variability of the connectome is larger than its sensitivity to the pathology then this will alter the accuracy of the experimental design study. Therefore one important analysis that we conducted here is assessing the variability of the connectome constructed from data acquired in the same imaging session, as well as after some period of time (one month) and compare it among connectomes constructed with different acquisitions schemes (DTI, HARDI and DSI) at different scales of parcellation (33-500). For simplicity, we show results for scale 33, since trends are kept across scales. Fig. 1 (a,b) shows that highest reproducibility is achieved within same day for DTI and DSI acquisitions (0.95, normalized network). At 1 month time difference, DTI shows the least correlation 0.76 . This shows that the connectivity maps can change in healthy subjects for about $20 \%$, within a month (worst case scenario performing DTI acquisition). HARDI shows the highest reproducibility across time, however this might be due to the small sample size. Same trend is observed for binarized connectomes, however the variability change is in the order of $15 \%$.

Intra-subject Structural Connectome Variability: To assess the differences between structural connectomes built using different modalities, we calculate the

\footnotetext{
2 https://sites.google.com/site/bctnet/

3 http://www.vsg3d.com/amira/overview
} 
similarity between different modalities within each subject. Fig. 11(c,d) demonstrates the biggest agreement in the connectomes between DTI and HARDI (both in normalized and binarized case). Taking the biggest agreement from the previous analysis as reference (same day DSI, normalized 0.95) we can observe that DSI and DTI connectomes disagree in about 23\%, DSI and HARDI disagree $19 \%$ whereas DTI and HARDI differ in 10\%. For binarized connectomes, the minimal agreement is between DSI and HARDI of about $23 \%$ difference (reference 0.75 ). These results should be taken with care given the low amount of subjects involved in the analysis.

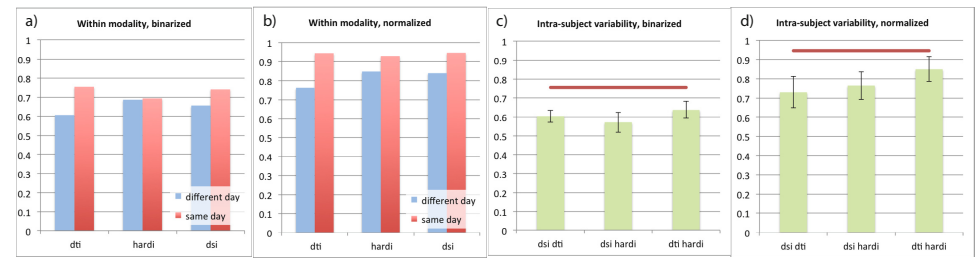

Fig. 1. left: Within subject, connectome variability with different modalities, for binarized (a) and normalized (b) networks. right: Correlation within intra-subject connectomes for binarized (c) and normalized (d) networks.

Network-Based Indices Subject Variability: Network-based indices are typically used to assert pathologies of the brain white matter. A very important fundamental analysis is to evaluate how sensitive and reproducible these indices are w.r.t. different acquisition modalities. As anatomical connectivity becomes increasingly sparse with higher scale, density values decrease. As we can observe in Fig. 2, DSI shows higher density values than DTI and HARDI, which may indicate a denser connectome. Global Efficiency is the average inverse shortest path length in the network. DSI presents higher global efficiency index, while DTI and HARDI show similar values. Characteristic path length is the average shortest path length in the network and it is normally used to compute small-worldness. It increases with scale, DSI showing the lowest values, while DTI and HARDI are similar. Strength is the average of the sum of weights of links connected to each node. It is seen as highly predictive of stronger functional

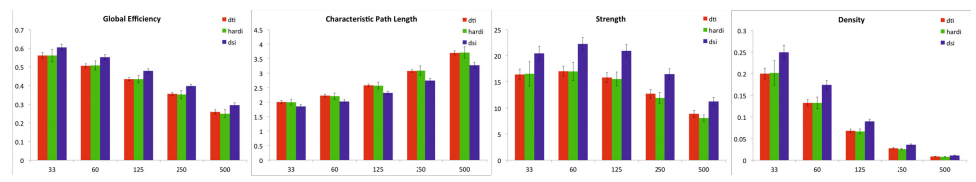

Fig. 2. Variability of network-based indices across scales and modality. Overall, DSI shows more distinctive indexes than DTI and HARDI (very similar to each other), however HARDI shows a higher standard deviation. This may suggest that HARDI is more sensitive to inter-subject variability. 


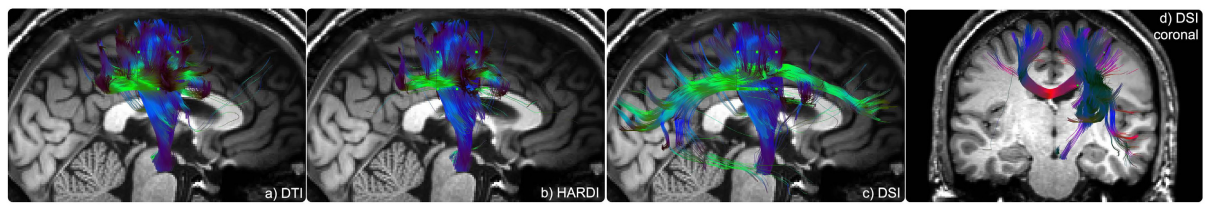

Fig. 3. Initial exploration of DTI vs HARDI vs DSI differences. DSI is obviously able to capture complex crossing structures, such as the superior longitudinal fasciculus connecting the frontal, occipital, parietal, and temporal lobes.
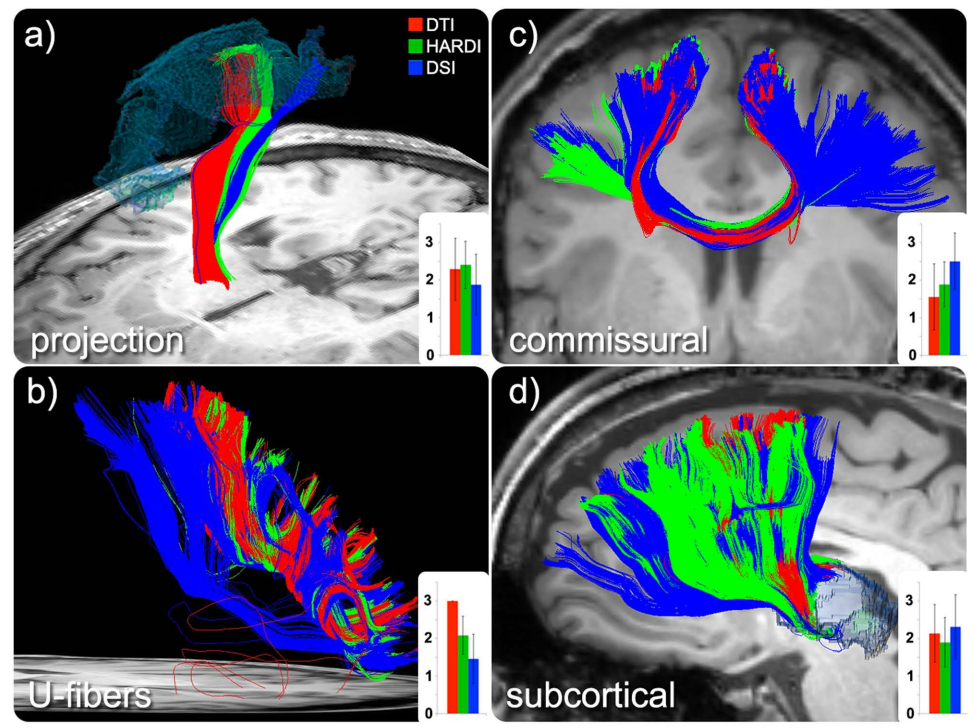

Fig. 4. Illustration of fibre bundle quality assessment. Red stands for DTI, green for HARDI and blue for DSI techniques. In each category the corresponding graphs show the average scores and standard deviation. a) DSI bundle originates in a different section of the postcentral medial ROI (likely the sensory leg nerves) while DTI and HARDI depict similar bundles, however HARDI bundle is more selective. b) Generally U-fibres are very well captured by DTI, whereas DSI does not depict the underlying anatomical shape, and often connects distant regions with additional long straight fibre bundles. HARDI generally has multiple isolated groups of U-fibre bundles. c) In the commissural bundles DSI is typically the best technique. It has long, extensive connections from the middle and upper parts of the cortex through the corpus callosum. DTI fibre bundles are vertically oriented, only connecting paramedial parts of this region. HARDI stands between the two techniques. d) These connections are composed of different fibre bundles connecting different regions of the thalamus with the cortex. In the evaluation some techniques proved better than others in relation to the anatomical correctness. Therefore in these regions when we perform the overall statistic we get similar reconstruction quality by all of the three techniques. 
connectivity. Same trend applies here, where DSI shows higher values while DTI and HARDI have similar behaviour. Higher strength values are shown in middles scales (60 and 125), suggesting a stronger depiction of the overall connectivity at these scales.

Track-Based Quality Assessment: Fig. 3 gives an illustration of the obvious benefits of DSI as a modelling and ultimately fibre reconstruction technique over DTI and HARDI since it is able to capture long fibre bundles such as the superior longitudinal fasciculus that is passing and crossing through complex fibre structures and many other complex configurations in the brain. This has been illustrated by Wedeen et al. [7]. However, in the connectomic approach with the predefined regions of parcellation we observe that these differences are not significantly favourable over DTI and HARDI. The differences have been clearly demonstrated in figure 4 where different techniques, for different fibre groups seem more anatomically correct. Furthermore, each technique missed the following fraction of fibre bundles (out of the analysed 40 bundles): DTI - 24\%, HARDI - $18 \%$, DSI - $14 \%$.

\section{Conclusion}

In this paper we have evaluated the characteristics and mutual differences of the structural connectomes constructed over different dwMRI acquisition schemes: DTI, HARDI and DSI. We have done this by employing graph based measures to real data that can quantify the information content and the differences between different techniques, at different scales of hierarchy. From these measures we observed that the connectome does not significantly capture richer information by using locally more accurate acquisition schemes such as DSI. In fact these techniques applied on a clinical scanner (3T) might produce noisier images leading to more aberrant fibres. Therefore in certain cases, such as the short U-fibres, simple techniques as DTI can outperform. Furthermore, in the case of global connectomic approaches, connectivity is typically measured as a function of the number of fibres passing through two ROIs of parcellation or average values of scalar measures such as FA. In certain approaches, the connectivity matrices are binarized, and as such the anatomical properties of the fibres are ignored. This explains the small differences (15-20\%) between the connectomes constructed by different acquisition schemes. In the qualitative analysis this is strongly demonstrated showing at times many aberrant fibres in DSI and HARDI approaches that in the connectivity matrices appear as valid connections. Future research should be aimed at capturing anatomical properties of the connectivity fibres as weights of the connectome, such as fibre volume, bundle cohesiveness and cluster based approaches. DSI can be seen as a powerful tool for neurosurgical application since it can detect complex fibre bundles, however it presents more aberrant fibers. In neurosurgery applications there is a direct visualization of the reconstructed fiber tracts, immediately identifiable by the neurosurgeons. However in connectomics, such false positives can be misleading (e.g., Fig. 4b) which implicates wrong connections between sub-regions at a higher scale. This can be 
wrongly interpreted as differences between two categories of subjects. Given the variation of performance in the qualitative analysis of the different reconstruction techniques, more modest (w.r.t. acquisition time and high b values), such as multi-shell HARDI approaches [12] would give significantly better results, combined with adaptive reconstruction techniques depending on the parts of the brain regions we evaluate. Finally, this study shows only preliminary results done on 5 subjects. To improve the statistic analysis, larger cohort of subjects should be analysed, and the intra-subject variability (20\% difference in longitudinal acquisition) must be taken into careful consideration.

\section{References}

1. Hagmann, P.: From Diffusion MRI to Brain Connectomics. PhD thesis, EPFL (2005)

2. Sporns, O., Tononi, G., Kötter, R., Ko, R.: The human connectome: A structural description of the human brain. PLoS Computational Biology 1(4), e42 (2005)

3. Bastiani, M., Shah, N.J., Goebel, R., Roebroeck, A.: Human cortical connectome reconstruction from diffusion weighted MRI: the effect of tractography algorithm. NeuroImage 62(3), 1732-1749 (2012)

4. Pierpaoli, C., Basser, P.J.: Toward a quantitative assessment of diffusion anisotropy. MRM 36, 893-906 (1996)

5. Tuch, D.S., Reese, T.G., Wiegell, M.R., Makris, N.G., Belliveau, J.W., Wedeen, V.J.: High Angular Resolution Diffusion Imaging Reveals Intravoxel White Matter Fiber Heterogeneity. MRM 48(4), 577-582 (2002)

6. Prčkovska, V., Roebroeck, A.F., Pullens, W.L.P.M., Vilanova, A., ter Haar Romeny, B.M.: Optimal Acquisition Schemes in High Angular Resolution Diffusion Weighted Imaging. In: Metaxas, D., Axel, L., Fichtinger, G., Székely, G. (eds.) MICCAI 2008, Part II. LNCS, vol. 5242, pp. 9-17. Springer, Heidelberg (2008)

7. Wedeen, V.J., Wang, R.P., Schmahmann, J.D., Benner, T., Tseng, W.Y.I., Dai, G., Pandya, D.N., Hagmann, P., D'Arceuil, H., de Crespigny, A.J.: Diffusion spectrum magnetic resonance imaging (DSI) tractography of crossing fibers. NeuroImage 41(4), 1267-1277 (2008)

8. Telesford, Q.K., Morgan, A.R., Hayasaka, S., Simpson, S.L., Barret, W., Kraft, R.A., Mozolic, J.L., Laurienti, P.J.: Reproducibility of Graph Metrics in fMRI Networks. Frontiers in Neuroinformatics 4, 10 (2010)

9. Daducci, A., Gerhard, S., Griffa, A., Lemkaddem, A., Cammoun, L., Gigandet, X., Meuli, R., Hagmann, P., Thiran, J.P.: The connectome mapper: an open-source processing pipeline to map connectomes with MRI. PloS one 7(12), e48121 (2012)

10. Wang, R., Benner, T., Sorensen, A.G., Wedeen, V.J.: Diffusion Toolkit: A Software Package for Diffusion Imaging Data Processing and Tractography. In: Proceedings of the 15th ISMRM Conference, p. 3720 (2007)

11. Butts, C.T.: Social network analysis: A methodological introduction. Asian Journal of Social Psychology 11(1), 13-41 (2008)

12. Zhan, L., Leow, A., Aganj, I., Lenglet, C., Sapiro, G., Yacoub, E., Harel, N., Toga, A., Thompson, P.: Differential information content in staggered multiple shell hardi measured by the tensor distribution function. I S Biomd. Imaging, 305-309 (March 30-April 2, 2011) 